\title{
0137 EPIDEMIOLOGY OF CHILD DROWNING INJURY IN THE PHILIPPINES
}

C A T Antonio, R J Consunji* Correspondence: Pasay City Health Office, Room 117 City Hall, F.B. Harrison Street, Pasay City 1302, Philippines

\subsection{6/ip.2010.029215.137}

A review and analysis of 40-years data on drowning injury (1963-2003) was conducted for this paper. On the average, at least 3000 Filipinos of all ages die annually from drowning injury $(0.43 \%$ of deaths for all age groups), more than a third $(35.6 \%)$ affect children under 14 years $(3.2 \%$ of deaths among children $0-14$ years). Mortality is highest among children ages 1-4 years compared to other age groups (Mortality Rate (MR) per $100000=6.4$ ), and among males compared to females for all age groups (MR 5.6). Drowning outranks traditional causes of child mortality (eg, meningitis, septicemia, malnutrition, tuberculosis and pneumonia), and will place 6 th, 3rd and 2 nd leading cause of mortality for children ages $1-4,5-9$ and 10-14 years, respectively. Through the study period, drowning mortality have remained largely unchanged, belying its under-recognition as a public health priority (MR1963=3.83; MR2003=3.48). The findings presented in this paper constitute only cases of reported fatal drowning, hence, these figures are to be taken as the minimum fatal drowning rates for the Philippines. There is also a paucity of local data on the circumstances surrounding drowning incidents, precluding identification of determinants, and consequently, the design of policies to address the burden of drowning in the country. These will be the subject of a subsequent report of Safe Kids Philippines, the Philippine Lifesaving Society, the Department of Health and the World Health Organisation. 